\title{
A Composite Phononic Crystal Design for Quasiparticle Lifetime Enhancement in Kinetic Inductance Detectors
}

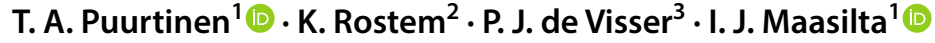 \\ Received: 3 August 2019 / Accepted: 21 February 2020 / Published online: 12 March 2020 \\ (c) The Author(s) 2020
}

\begin{abstract}
A nanoscale phononic crystal filter (reflector) is designed for a kinetic inductance detector where the reflection band is matched to the quasiparticle recombination phonons with the aim to increase quasiparticle lifetime in the superconducting resonator. The inductor is enclosed by a $1-\mu \mathrm{m}$-wide phononic crystal membrane section with two simple hole patterns that each contain a partial spectral gap for various high-frequency phonon modes. The phononic crystal is narrow enough for low-frequency thermal phonons to propagate unimpeded. With 3D phonon scattering simulation over a $40 \mathrm{~dB}$ attenuation in transmitted power is found for the crystal, which is estimated to give a lifetime enhancement of nearly two orders of magnitude.
\end{abstract}

Keywords Phononic crystal · Kinetic inductance detector · Finite element method · Phonon scattering

\section{Introduction}

Phononic crystals (PnCs) are periodic structures designed to modify properties of elastic and acoustic waves by the Bragg scattering mechanism or by localized resonances [1]. Applications have been proposed in many engineering and physics areas, including vibration and noise absorption, acoustic cloaking and metamaterials research [2]. Recently, with the ability to fabricate microscopic phononic crystals, thermal applications have also appeared benefiting from the wave interference of phonons at thermal wavelengths. This could be utilized for instance in improving thermoelectric devices by reduction in thermal conductivity, guiding and focusing

I. J. Maasilta

maasilta@jyu.fi

1 Department of Physics, Nanoscience Center, University of Jyväskylä, 40014 University of Jyväskylä, Finland

2 NASA Goddard Space Flight Center, 8800 Greenbelt Road, Greenbelt, MD 20771, USA

3 SRON, Netherlands Institute for Space Research, Sorbonnelaan 2, 3584 Utrecht, CA, The Netherlands 
heat in nanostructures and using heat in phononic information processing [3-5]. At sub-Kelvin temperatures, phononic crystals fabricated in a suspended membrane have been shown to reduce thermal conductance by several orders of magnitude [6, 7], which is a key to improve sensitivity in many low-temperature bolometric detector technologies.

Kinetic inductance detectors (KID), on the other hand, are extremely sensitive non-bolometric photon detectors based on superconducting microwave resonators [8]. The operation principle of a KID is to break Cooper pairs into quasiparticles in a superconducting microwave resonator with signal photons, thus changing the impedance of the resonator, which can be measured with a coupled microwave readout line. Quasiparticles recombine quickly back into Cooper pairs by emitting phonons at frequencies $\geq 2 \Delta / h$, corresponding to the energy gap of the superconducting material. The recombination phonons can break other Cooper pairs, but phonons escaping the system result in a lost signal limiting the responsivity of the detector. Simply removing material around the resonator does not help because a good thermal contact is desired so that low-frequency phonons due to dissipation of the microwave readout signal can escape. Recently, Rostem et al. presented calculations for an effective recombination lifetime enhancement of quasiparticles in a phononic crystal isolated kinetic inductance detector (PhiKID) [9]. They showed how a PnC notch filter with a $0.3 \Delta / h$ wide reflection band and a $40 \mathrm{~dB}$ attenuation positioned slightly off from the gap frequency $2 \Delta / h$ of a superconductor can increase the lifetime by two orders of magnitude.

A number of publications in the field of ultrasound physics discuss the formation of band gaps or scattering of elastic waves in plates with obstacles [10-12], which are useful starting points in finding suitable PnC geometries with full band gaps for the recombination phonons. However, it will be difficult to fabricate such PnCs for the most commonly used low- $T_{\mathrm{c}}$ superconductors, such as aluminum, which has $2 \Delta / h \sim 89 \mathrm{GHz}$. For example, creating a matched reflection band for aluminum with a thru-hole pattern in a suspended silicon nitride membrane would steer the fabrication toward very thin membranes $(\sim 33 \mathrm{~nm})$ and the smallest features in a few nanometer length scale [6]. Even for thin-film hafnium, which has $2 \Delta / h \sim 32 \mathrm{GHz}$, a matching $\mathrm{PnC}$ design with a thru-hole pattern would require a 95 -nm-thick membrane with a distance between holes only $5 \mathrm{~nm}$.

In this paper, we propose a simulation-based design for a composite $\mathrm{PnC}$ consisting of two simple hole patterns with minimum feature size $50 \mathrm{~nm}$, and a relatively low combined width of $1 \mu \mathrm{m}$. Each of the patterns possess partial spectral gaps for various modes, which effectively create a full reflection band for highfrequency athermal recombination phonons, while thermal low-frequency phonons pass through the structure unimpeded. Interestingly, a PnC will also reject athermal phonons released during the energy downconversion of quasiparticles excited to energies significantly greater than $\Delta$. The downconversion process releases phonons at the scale of the Debye frequency of the metal. The loss of these so-called hot phonons from the superconductor to the bath is known to limit the performance of many cryogenic detectors, including STJs [13], TESs [14] and KIDs [15]. A PnC can effectively recycle hot short wavelength phonons through a purely geometric scattering mechanism from the hole walls, due to the lack of material that is etched 
away. At the same time, due to the scale of the PnC pattern, large wavelength thermal phonons with frequencies below $10 \mathrm{GHz}$ have unity transmission. Thus, a PnC may be effective at improving the performance of KIDs that utilize aluminum, or other metals with similar $T_{\mathrm{c}}$ values, to detect visible and near-infrared photons.

\section{Scattering Simulations}

Phonon propagation and scattering in PnC membranes at sub-Kelvin temperatures is described with a 3D continuum linear elasticity theory [16]. For smooth membranes, i.e., where the surface roughness is small compared to the phonon wavelength, phonon mean free paths have been measured to exceed $100 \mu \mathrm{m}$ [17], thus phonons can be treated as ballistic waves that can only scatter from the PnC structure. The membrane material can be assumed to be isotropic and homogeneous, and it is thus characterized by a constant density $\rho$ and two independent elastic material constants, often denoted by the Lame parameters $\lambda$ and $\mu$. The displacement field $U=(u, v, w)$ in such system obeys

$$
\lambda \Delta U+(\lambda+\mu) \nabla \cdot \operatorname{div} U+\rho F=\rho \frac{\partial^{2} U}{\partial t^{2}}, \quad \text { in } \Omega
$$

where $\Omega$ is the domain describing the infinite PnC membrane geometry (see Fig. 1 left) and $F=F(x, y, z, t)$ is the driving force applied in the domain. On the domain boundaries (the top and bottom surfaces, and hole boundaries), we apply the stressfree boundary condition $\hat{n} \cdot \sigma(U)=0$. Here, $\sigma(\cdot)=\left(\sigma_{i j}(\cdot)\right), i, j=x, y, z$ is the stress tensor and $\hat{n}$ is the surface normal vector. Assuming time harmonic force $F$ and solution $U$, the derivative $\partial^{2} / \partial t^{2}$ can be replaced by $-\omega^{2}$, where $\omega$ is the angular frequency of the force [16].

The time harmonic problem of Eq. (1) is solved in the frequency domain using the finite element method and COMSOL Multiphysics v5.3 software. When the incident wave strikes the $\mathrm{PnC}$ from a $90^{\circ}$ angle (Fig. 1 left), the scattered field is periodic in $\Omega$ in the $y$-direction, and thus the infinite domain $\Omega$ can be truncated as shown in Fig. 1 (right). Periodic boundary conditions for the solution $U$ are set on the $x-z$ boundaries so that unphysical coupling or conversion of the modes does not

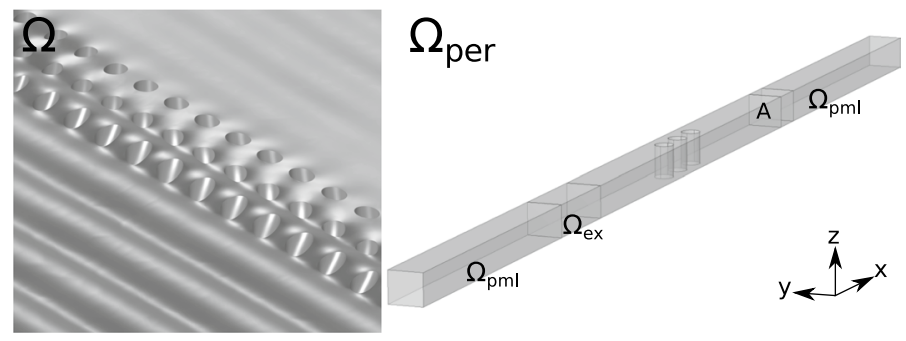

Fig. 1 (Color online) Left: 3D simulation of Lamb wave scattering in an infinite phononic crystal membrane. Right: Truncated periodic subdomain $\Omega_{\text {per }}$ of the membrane. An incident phonon is excited at the $\Omega_{\mathrm{ex}}$ region, and the scattered field from the crystal is absorbed in perfectly matched layers $\Omega_{\mathrm{pml}}$ 
occur there. Finally, perfectly matched layers $\Omega_{\mathrm{pml}}$ are created at the ends to absorb the scattered phonons. We remark that it is in principle possible to simulate the system in a full-scale 3D model without periodicity allowing variable incident angles, but this would greatly increase the computational cost.

To study reflection and mode conversion properties of PnCs, we aim to run the scattering simulations with pure shear and Lamb modes [16], which are the solutions for Eq. (1) in the regions far away from the PnC. However, a typical problem with excitation of pure modes is that the scattered field re-scatters from the emitter and thus interferes with the original incident phonon. Neglecting the interference can lead to incorrect evaluation of transmission and reflection coefficients. We solved this problem by taking advantage of the biorthogonality property of the modes in the force term $F$ applied in the 3D subdomain $\Omega_{\mathrm{ex}}[18,19]$. If the solution $U$ on $\Omega_{\text {ex }}$ is expressed as $U=U_{\text {inc }}^{+}+U_{\text {scat }}$, where $U_{\text {scat }}=\sum_{j} \alpha_{j} U_{j}^{-}$and plus (minus) denotes waves propagating in the positive (negative) $x$-direction, then by the biorthogonality property

$$
\int_{\Omega_{\mathrm{ex}}} U_{j}^{-} \cdot \overline{\sigma_{x}\left(U_{\mathrm{inc}}^{+}\right)} \mathrm{d} V-\int_{\Omega_{\mathrm{ex}}} \overline{U_{\text {inc }}^{+}} \cdot \sigma_{x}\left(U_{j}^{-}\right) \mathrm{d} V=0
$$

for all mode indices $j$. Here, $\sigma_{x}(\cdot):=\left(\sigma_{x x}(\cdot), \sigma_{x y}(\cdot), \sigma_{x z}(\cdot)\right)^{\mathrm{T}}$ is a vector of the stress tensor components in the $x$-direction, and the overline denotes the complex conjugate. In the weak formulation of the elasticity equation (1) (see e.g., [20]), the domain force term appears in the same form as the first term of the biorthogonality property (2), if the force term is set to $F=\overline{\sigma_{x}\left(U_{\text {inc }}^{+}\right)}$and the functions $U_{j}^{-}$are replaced by the finite element basis functions. We then add another integral term in the weak formulation corresponding to the second term of the biorthogonality property, which together with the first term guarantees that all scattered wave components $U_{j}^{-}$in the solution $U$ result to zero in these integrals and thus cannot backscatter from the subdomain $\Omega_{\text {ex }}$. Phonon modes $U_{j}^{ \pm}$in the membrane can be calculated either numerically, or semi-analytically by solving the Rayleigh-Lamb dispersion equations [16]. After normalization, functions $U_{j}^{ \pm}$are inserted into the weak formulation integrals for a numerical solution of the scattering problem.

Similarly, the phonon transmission power can be evaluated by the biorthogonality relation [19]. Total mechanical power of the elastic field $U$ through a boundary $A$ can be expressed as the sum of the single mode $U_{j}^{+}$contributions $P_{j}$, where

$$
P_{j}=\Re\left[\frac{i \omega}{4} \int_{A}\left(U \cdot \overline{\sigma_{x}\left(U_{j}^{+}\right)}-\overline{U_{j}^{+}} \cdot \sigma_{x}(U)\right) \mathrm{d} A\right] .
$$

\section{Results}

To find a practical design for the PnC membrane with good reflective properties in the $32 \mathrm{GHz}$ region (corresponding to the energy gap of thin-film hafnium), we numerically calculated phonon dispersion relations for several simple infinite 
cylindrical hole patterns in crystalline silicon membranes varying the lattice constant, hole filling fraction, membrane thickness and the lattice geometry. The designs with a full spectral gap at $32 \mathrm{GHz}$ found this way were not feasible for fabrication with current lithography processes because of too small feature sizes going below 5-nm length scale. A full spectral gap, however, is not mandatory, because a similar effect can be achieved by combining several simpler structures in series each possessing a partial spectral gap for some Lamb modes. With the scattering simulation, we can then estimate the effect of the composite structure for each phonon mode.

The most practical geometry found, shown in Fig. 2, is a composite thru-hole PnC design, which functions as a phononic multi-pole filter [21]. The geometry contains cylindrical holes in hexagonal and square lattices in a thin silicon membrane with thickness of $100 \mathrm{~nm}$. The lattice constant for both lattices is $110 \mathrm{~nm}$ and the hole diameter is $60 \mathrm{~nm}$. The square lattice is 5 cells and the hexagonal lattice 5 cells wide making the composite crystal only about $1 \mu \mathrm{m}$ wide with the smallest feature (neck width) $50 \mathrm{~nm}$. The corresponding dispersion spectra are also shown for each of the components. This geometry can be fabricated with a single exposure etch process.

It is immediately seen from the dispersion relations that at the low-frequency (sub-10 GHz) range, the spectra resemble a full-membrane spectrum [16], thus the PnC should have minimal effect on thermal conductance in the membrane. In addition, neither of the lattices seem to have a full spectral gap at $32 \mathrm{GHz}$. However, further analysis of the modes reveals partial spectral gaps that can be utilized for the same effect. We remark that pure Lamb modes do not exist in the PnCs, but in these lattices (anti-)symmetrically polarized incident waves are likely to remain (anti-) symmetric also in the PnC. In other words, if there are no propagating antisymmetric out-of-plane modes in the $\mathrm{PnC}$, then it can be expected that incident antisymmetric out-of-plane Lamb modes are reflected from the PnC. To locate the partial

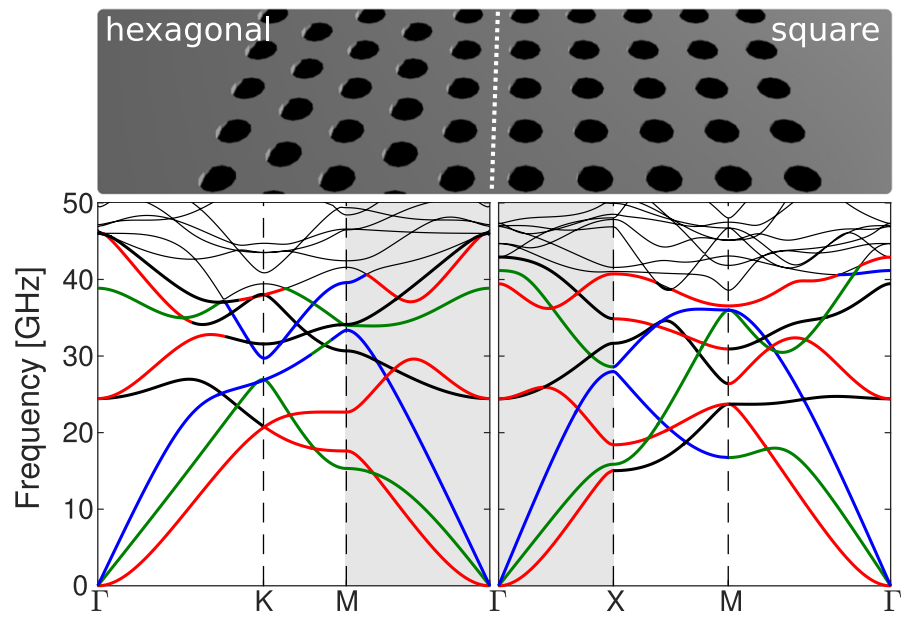

Fig. 2 (Color online) Top: A composite phononic crystal structure containing cylindrical holes in square and hexagonal lattice configurations. Left: Spectrum of the hexagonal lattice. Right: Spectrum of the square lattice. For color coding, see text 
spectral gaps in Fig. 2, we classified the modes based on a component analysis of the fields $U$. In Fig. 2, red corresponds to the (mainly) antisymmetric out-of-plane mode, green is the in-plane shear, blue is the symmetric out-of-plane mode, and black is the second-order antisymmetric out-of-plane mode. The gray highlighted area shows the direction perpendicular to the $\mathrm{PnC}$, which can be simulated with the scattering model. Following the colored curves, partial spectral gaps can be identified for each of the mode types in this region.

Finally, the scattering simulation is used to calculate the attenuation level of the composite structure. In Fig. 3, the transmitted power of each of the propagating Lamb and horizontal shear modes is shown around the $32-\mathrm{GHz}$ frequency region. Colors of the various curves denote different mode polarizations, and the incident mode color is shown at the top-left corner. For all modes, a fairly wide reflection band is created at $32 \mathrm{GHz}$, which is highlighted by blue. Cut-off bands for the second-order modes are highlighted by red, which means that these modes do not exist at low-frequency range. Incident modes in most cases forward-scatter to the same mode, but a small amount of coupling can be identified for the first- and the second-order antisymmetric out-of-plane modes as revealed by the secondary curves in those plots. We remark that changing the incident angle slightly will not cause significant qualitative changes to the transmitted power in Fig. 3, and similar attenuation levels and band locations are likely to exist for a range of incident angles.

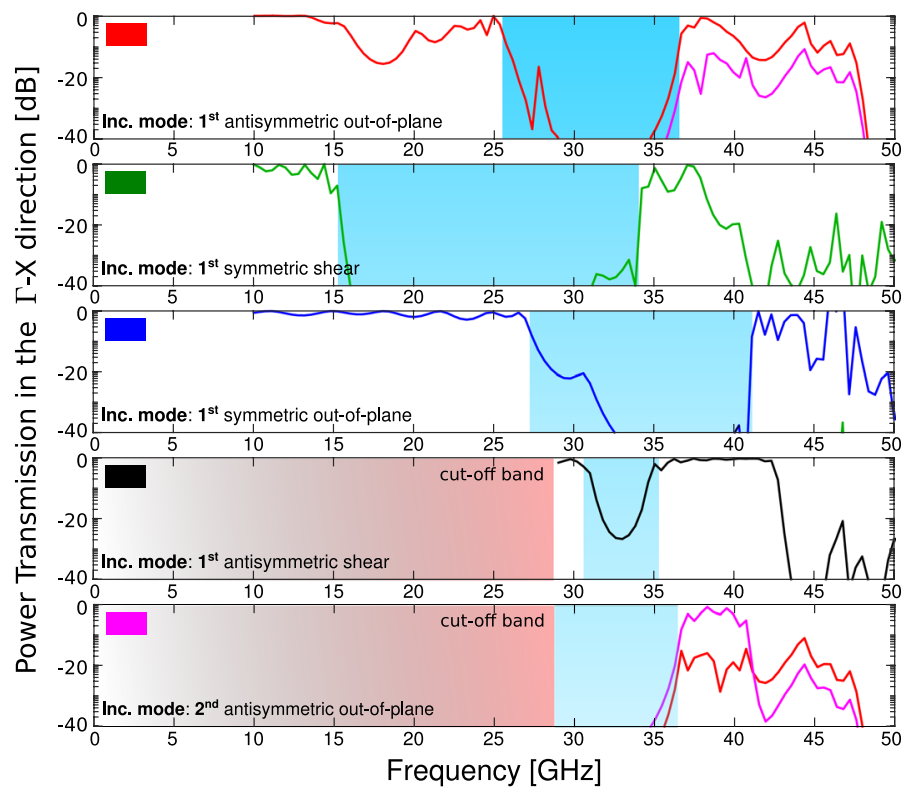

Fig. 3 (Color online) Transmitted phonon power through the composite phononic crystal of Fig 2 with normal incidence. Colored curves in the plots reveal the amount of coupling: e.g., in the first plot, the first antisymmetric out-of-plane mode (red) couples with the second-order mode (magenta) at $f>35 \mathrm{GHz}$. For all modes, there exists a significant reflection band at $32 \mathrm{GHz}$, while at the low frequency limit, power transmission approaches unity leading to good thermal conductance 
Effective quasiparticle lifetime enhancement for each phonon mode can be calculated by inserting reflection band widths and locations for each of curves in Fig. 3 into the equations in Rostem et al. [9]. A single effective reflection band location and width parameters for the total power cannot be formulated from our data, because the emitted phonon angular distribution and population in various mode types were not fully analyzed. In a PhiKID detector, however, the antisymmetric modes are likely to be dominant, because the metal thin-film emitter is located only on the top-side of the suspended membrane, thus making the source geometry antisymmetric relative to the membrane. Thus, for an estimate of the total effective lifetime enhancement, the first antisymmetric mode band gap position $32 \mathrm{GHz}(=2 \Delta / h)$ and width $5 \mathrm{GHz}(=0.3 \Delta / h)$ are shown in Fig. 3, and then the lifetime enhancement factor $\chi$ can be read from Rostem et al. [9] as shown in Fig. 5. With these numbers, the value of $\chi$ approaches $10^{2}$ indicating roughly two orders of magnitude increase in quasiparticle lifetime.

\section{Conclusion}

Nanoscale phononic crystals (PnCs) appear as promising functional components in many low temperature detector technologies. Kinetic inductance detectors have been suggested to benefit from notch filter-type PnCs when the filter is tuned to the quasiparticle recombination phonon frequencies of the KID superconducting resonator. However, for many superconductor materials, fabricating such a filter is challenging even with state-of-the-art nanofabrication techniques, because of extremely small features required for the PnC. Using 3D elastic wave scattering simulations, we demonstrated how a composite PnC assembled from two simple hole patterns can reach at least $40 \mathrm{~dB}$ power attenuation level for the recombination phonons incident from the perpendicular direction, which has been estimated to result in effective quasiparticle lifetime enhancement of two orders of magnitude. The smallest feature in this design was only $50 \mathrm{~nm}$ and the structure was only $1 \mu \mathrm{m}$ wide making it relatively simple to fabricate with modern electron beam lithography techniques. Based on these results, a phononic crystal isolated kinetic inductance detector could be realized in the near future.

Acknowledgements Open access funding provided by University of Jyväskylä. This study was supported by the Academy of Finland Project Number 298667. K. Rostem gratefully acknowledges financial support from a NASA Astrophysics Research and Analysis Grant (NNX17AH83G). P. J. de Visser was financially supported by the Netherlands Organisation for Scientific Research NWO (Veni Grant 639.041.750).

Open Access This article is licensed under a Creative Commons Attribution 4.0 International License, which permits use, sharing, adaptation, distribution and reproduction in any medium or format, as long as you give appropriate credit to the original author(s) and the source, provide a link to the Creative Commons licence, and indicate if changes were made. The images or other third party material in this article are included in the article's Creative Commons licence, unless indicated otherwise in a credit line to the material. If material is not included in the article's Creative Commons licence and your intended use is not permitted by statutory regulation or exceeds the permitted use, you will need to obtain permission directly from the copyright holder. To view a copy of this licence, visit http://creativecommons.org/licen ses/by/4.0/. 


\section{References}

1. M.S. Kushwaha, P. Halevi, L. Dobrzynski, B. Djafari-Rouhani, Phys. Rev. Lett. 71, 2022 (1993). https://doi.org/10.1103/PhysRevLett.71.2022

2. R.V. Craster, S. Guenneau (eds.), Acoustic Metamaterials, vol. 166 of Springer Series in Material Science (Springer, Berlin, 2013)

3. A.J. Minnich, M.S. Dresselhaus, Z.F. Ren, G. Chen, Energy Environ. Sci. 2, 466-479 (2009). https ://doi.org/10.1039/B822664B

4. R. Anufriev, A. Ramiere, J. Maire, M. Nomura, Nat. Commun. 8, 15505 (2017). https://doi. org/10.1038/ncomms 15505

5. N. Li, J. Ren, L. Wang, P. Hänggi, B. Li, Rev. Mod. Phys. 84, 1045 (2012). https://doi.org/10.1103/ RevModPhys.84.1045

6. N. Zen, T.A. Puurtinen, T.J. Isotalo, S. Chaudhuri, I.J. Maasilta, Nat. Commun. 5, 3435 (2014). https://doi.org/10.1038/ncomms4435

7. Y. Tian, T.A. Puurtinen, Z. Geng, I.J. Maasilta, Phys. Rev. Appl. 12, 014008 (2019). https://doi. org/10.1103/PhysRevApplied.12.014008

8. P.K. Day, H.G. LeDuc, B.A. Martin, A. Vayonakis, J. Zmuidzinas, Nature 425, 817 (2003). https:// doi.org/10.1038/nature02037

9. K. Rostem, P.J. de Visser, E.J. Wollack, Phys. Rev. B 98, 014522 (2018). https://doi.org/10.1103/ PhysRevB.98.014522

10. T. Gorishnyy, C.K. Ullal, M. Maldovan, G. Fytas, E.L. Thomas, Phys. Rev. Lett. 94, 115501 (2005). https://doi.org/10.1103/PhysRevLett.94.115501

11. Y. Pennec, B. Djafari-Rouhani, H. Larabi, J.O. Vasseur, A.C. Hladky-Hennion, Phys. Rev. B 78, 104105 (2008). https://doi.org/10.1103/PhysRevB.78.104105

12. R.P. Moiseyenko, S. Herbison, N.F. Declercq, V. Laude, J. Appl. Phys. 11, 034907 (2012). https:// doi.org/10.1063/1.3682113

13. D.D.E. Martin, P. Verhoeve, A. Peacock, Appl. Phys. Lett. 88, 123510 (2006). https://doi. org/10.1063/1.2187444

14. A.G. Kozorezov, J.K. Wigmore, Appl. Phys. Lett. 89, 223510 (2006). https://doi. org/10.1063/1.2397016

15. W. Guo et al., Appl. Phys. Lett. 110, 212601 (2017). https://doi.org/10.1063/1.4984134

16. K.F. Graff, Wave Motion in Elastic Solids (Dover, New York, 1991)

17. H.F.C. Hoevers, M.L. Ridder, A. Germeau, M.P. Bruijn, P.A.J. de Korte, R.J. Wiegerink, Appl. Phys. Lett. 86, 251903 (2005). https://doi.org/10.1063/1.1949269

18. R.D. Gregory, J. Elast. 13, 351-355 (1983). https://doi.org/10.1007/BF00043002

19. A. Gunawan, S. Hirose, J. Acoust. Soc. Am. 115, 3 (2004). https://doi.org/10.1121/1.1639330

20. T.J.R. Hughes, The Finite Element Method: Linear Static and Dynamic Finite Element Analysis (Dover, New York, 2000)

21. K. Rostem, D.T. Chuss, K.L. Denis, E.J. Wollack, J. Phys. D Appl. Phys. 49, 25 (2015). https://doi. org/10.1088/0022-3727/49/25/255301

Publisher's Note Springer Nature remains neutral with regard to jurisdictional claims in published maps and institutional affiliations. 